\title{
The problem of the Pleiades distance
}

\section{Constraints from Strömgren photometry of nearby field stars}

\author{
D. Stello and P. E. Nissen \\ Institute of Physics and Astronomy (IFA), University of Aarhus, 8000 Aarhus, Denmark \\ e-mail: pen@ifa.au.dk
}

Received 3 April 2001 / Accepted 7 May 2001

\begin{abstract}
The discrepancy between the Pleiades cluster distance based on Hipparcos parallaxes and main sequence fitting is investigated on the basis of Strömgren photometry of F-type stars. Field stars with the same metallicity as the Pleiades have been selected from the $m_{1}$ index and a technique has been developed to locate the ZAMS of these field stars in color-magnitude diagrams based on the color/temperature indices $b-y, v-y$, and $\beta$. Fitting the Pleiades to these ZAMS relations results in a distance modulus of $5.61 \pm 0.03$ mag in contrast to the Hipparcos modulus of $5.36 \pm 0.06 \mathrm{mag}$. Hence, we cannot confirm the recent claim by Grenon (1999) that the distance problem is solved by adopting a low metallicity of the Pleiades $\left([\mathrm{Fe} / \mathrm{H}]_{\text {Pleiades }}=-0.11\right)$ as determined from Geneva photometry. The metallicity sensitivity of the ZAMS determined by the field stars is investigated, and by combining this sensitivity in all three color/temperature indices $b-y, v-y$, and $\beta$ we get a independent test of the Pleiades distance modulus which support our value of $5.61 \mathrm{mag}$. Furthermore, the field star sample used for the comparison is tested against theoretical isochrones of different ages to show that evolutionary effects in the field star sample are not biasing our distance modulus estimate significantly. Possible explanations of the Pleiades distance problem are discussed and it is suggested that the discrepancy in the derived moduli may be linked to a non-spherical shape of the cluster.
\end{abstract}

Key words. open clusters and associations: individual: Pleiades - stars: Hertzsprung-Russell (HR) and C-M diagrams - stars: distances - stars: evolution - stars: abundances

\section{Introduction}

The Hipparcos Space Astrometry Mission has provided accurate absolute trigonometric parallaxes for roughly 120000 stars, which are distributed all over the sky, and hence relatively accurate distance measurements for stars at a much larger distance than previous obtainable from ground based observations. This has given the opportunity to compare distances of several open clusters derived from direct trigonometric measurements with those derived from main sequence (MS) fitting. For some of the clusters there are discrepancies between the derived distances, but in most cases the differences are within the estimated uncertainties. An exception is the Pleiades for which the distance modulus derived using the mean of the Hipparcos parallaxes is almost $0.3 \mathrm{mag}$ smaller than that derived using the MS fitting technique. Possible

Send offprint requests to: D. Stello, e-mail: stello@ifa.au.dk explanations of this anomaly are:

1. The errors of the MS fitting technique may be underestimated. This could arise from difficulties in the technique itself, or it could be due to serious errors in the adopted chemical composition of the Pleiades cluster.

2. There may be systematic errors on small angular scales in the sky of the Hipparcos parallaxes which are underestimated. This could bias the inferred distance to clusters that only cover a small angular area in the sky.

If these possibilities can be excluded we may have to draw the important conclusion that the theory of stellar structure and evolution is incomplete or in other words: the Vogt-Russell theorem that the location of a star in the Hertzsprung-Russell diagram is uniquely determined by its mass, age, and composition is violated. It is this aspect of the Pleiades distance problem that makes it so interesting.

The mean parallax of the Pleiades cluster inferred from the Hipparcos data ranges from $8.60 \pm 0.24$ mas (Mermilliod et al. 1997) to $8.45 \pm 0.25$ mas (van Leeuwen 1999). These parallaxes correspond to a distance 
interval of $116 \pm 3 \mathrm{pc}$ to $118 \pm 4 \mathrm{pc}$ or a distance modulus interval of $5.33 \pm 0.06 \mathrm{mag}$ to $5.37 \pm 0.07 \mathrm{mag}$. These distance moduli should be compared with those found from the MS fitting method. Pinsonneault et al. (1998) find a distance modulus of $5.60 \pm 0.05 \mathrm{mag}$, based on an extensive multi color MS fitting analysis. They make use of several open clusters to check for different possible error sources, and both isochrones and an empirical Hyades MS are used as the zero point of the ZAMS. Pinsonneault et al. (1998) suggest that the discrepancy between the results from Hipparcos and the MS fitting method is due to spatial systematic errors on small angular scales in the Hipparcos data (Pinsonneault et al. 1998, Fig. 20) which are larger than expected (Lindegren 1988, 1989, 1997). From a comparison of the Pleiades MS with those of the Hyades and $\alpha$ Persei clusters Eggen (1998) also concludes that the Hipparcos parallax distance of the Pleiades may be in error by some $10 \%$. An investigation of the possible spatial systematic errors in the Hipparcos data is performed by Narayanan \& Gould (1999) who confirm the distance modulus of around $5.6 \mathrm{mag}$ by an estimate of $5.58 \mathrm{mag}$, though with a fairly large error of $\pm 0.18 \mathrm{mag}$. Their investigation is based on a variant of the moving cluster method to get distances for each individual cluster member. On the basis of the stated spatial systematic errors in the Hipparcos data Robichon et al. (1999) make an extensive investigation of this problem, which makes use of the method of analyzing Hipparcos intermediate data described by van Leeuwen \& Evans (1998). They recalculate the Hipparcos parallaxes and find a distance modulus of $5.36 \pm 0.06 \mathrm{mag}$ for the Pleiades which is the value adopted in this paper for comparisons.

Recently, Grenon (1999) has claimed that the Pleiades distance problem is solved by adopting a cluster metallicity of $[\mathrm{Fe} / \mathrm{H}]_{\text {Pleiades }}=-0.11$ as determined from Geneva photometry instead of $[\mathrm{Fe} / \mathrm{H}]_{\text {Pleiades }} \simeq 0.0$ as determined from high resolution spectroscopy (Boesgaard \& Friel 1990). In particular, Geneva photometry points to a large difference in the metallicity of Praesepe and Pleiades $(\Delta[\mathrm{Fe} / \mathrm{H}]=0.28)$. To a large extent this explains the offset between the two clusters in various color-magnitude diagrams (Robichon et al. 2000).

The purpose of the current study is to investigate the discrepancy between the Pleiades distance inferred from the Hipparcos mean parallax and from the MS fitting method by comparing Strömgren uvby $-\beta$ photometry of Pleiades F-type stars with field stars having the same metallicity as the Pleiades. We start out with a presentation of the Pleiades cluster and field star data followed by the calibration formulas and selection of stars used for the rest of the reductions. Then the MS fitting analysis is described including the fitting technique, which has been developed to locate the ZAMS of the field star sample, and how we test the age range of the field stars. Furthermore, a metallicity sensitivity analysis is presented and finally we discuss possible answers to the Pleiades problem including the suggestion that the discrepancy in the derived distance moduli could be a real effect caused by the structure of the cluster.

\section{MS fitting with Strömgren photometry}

The Pleiades, shifted to the distance inferred from the Hipparcos mean parallax, are compared to nearby field stars of the same metallicity in a color-magnitude diagram. This is done to see if the ZAMS defined by the field stars (hereafter denoted $\mathrm{ZAMS}_{\text {Field }}$ ) does coincide with the Pleiades MS. Further, the distance modulus of the Pleiades is inferred from a best fit by the comparison of the Pleiades MS and the ZAMS Field. This investigation also includes a sensitivity analysis of the $\mathrm{ZAMS}_{\text {Field }}$ locus to changes in $[\mathrm{Fe} / \mathrm{H}]$. The analysis is performed for F-type stars, and in three color/temperature indices $b-y, v-y$, and $\beta$, to check for robustness and possible hidden errors in the MS fitting technique.

The advantage of using field stars to define the zero point of the distance modulus is that the investigation will be purely empirical, and not affected by some possible lacking ingredients in the theoretical calculations of the isochrones. So this investigation only relies on the VogtRussell theorem (Sect. 1). The shape of the Pleiades MS turns out to be very similar to the $Z_{A M S}$ Field shape and hence we avoid the problem of fitting to isochrones which do not match the cluster MS at every temperature interval. Using F-type stars gives the opportunity to correct for interstellar reddening and to estimate $[\mathrm{Fe} / \mathrm{H}]$, based on the Strömgren indices and available empirical calibration formulas. The assumed shape of the $\mathrm{ZAMS}_{\text {Field }}$ used for the comparison between the Pleiades MS and the field stars is found by a second-order robust least squares fit to the Pleiades stars.

\subsection{Data}

The $u v b y-\beta$ data for the Pleiades is taken from Table II of Crawford \& Perry (1976) (hereafter CP76), which contains members classified as F-type stars by CP76. The mean error of one observation, was determined from the internal scatter in the measurements of all the Pleiades stars (Table I CP76), and were given as follows, $\sigma(\beta)=0.011$, $\sigma(b-y)=0.009, \sigma\left(m_{1}\right)=0.011$, and $\sigma\left(c_{1}\right)=0.012$ (CP76). Taking into account that each star has been observed about 5 times we obtain the following typical mean errors of the mean indices of one star: $\sigma(\beta)=0.005$, $\sigma(b-y)=0.004, \sigma\left(m_{1}\right)=0.005$, and $\sigma\left(c_{1}\right)=0.005 \mathrm{mag}$.

The magnitudes of the stars in CP76 were adopted from Johnson \& Mitchell (1958) (based on the $U B V$ system), so to get the Strömgren values ( $y$ transformed to $V$ ) the star magnitudes are taken from the WEBDA database (Mermilliod 2000). For about half of the stars the Strömgren value is not available, and the magnitudes are taken to be the average of the $V$ values from $U B V$ observations (also given in the WEBDA database). It is noted that for stars with both uvby and $U B V$ photometry, the $V$ magnitudes agree within $\pm 0.02 \mathrm{mag}$. 
The field star data has been taken from a catalogue of $\sim 30000$ stars observed in uvby $-\beta$ (Olsen 1999). This catalogue has been made by merging five published catalogues; all by E. H. Olsen. The sample used in this investigation (F-type stars) is based on three of these catalogues (Olsen 1983, 1988, 1994). The overall $\mathrm{rms}$ internal error of one observation is $\sigma(V)=0.005, \sigma(b-y)=0.004$, $\sigma\left(m_{1}\right)=0.006, \sigma\left(c_{1}\right)=0.007$, and $\sigma(\beta)=0.007$. These errors are the conservative ones, in some of the catalogues they were in fact $\sim 0.002 \mathrm{mag}$ smaller, but the conservative ones are adopted in this investigation. The majority of the stars were observed only once and a few 2 or 3 times, so the errors in the mean photometric values per star are not significantly different from the errors stated above.

The absolute magnitudes are derived using the reddening corrected apparent magnitudes $V_{0}$ (transformed from y), and the Hipparcos parallaxes (ESA 1997). The mentioned possible spatial systematic error in the Hipparcos parallaxes does not affect the locus of the $\mathrm{ZAMS}_{\text {Field }}$, because it can only have effects on small angular scales, and the field stars are distributed "randomly" all over the sky. Thus the error contributions from the parallaxes in the zero point for the $\mathrm{ZAMS}_{\text {Field }}$ is the global parallax error, which is less than 0.1 mas (Arenou et al. 1997).

In addition to the internal errors for the Pleiades photometry by CP76 and for the field stars by Olsen (1999) there could be systematic differences between the two sources. Especially the $\beta$ index is critical, because the reddening and hence the corrected color indices $(b-y)_{0}$ and $(v-y)_{0}$ is determined from $\beta$ (Sect. 2.2.1). It is, however, very unlikely that systematic errors in $\beta$ could be large enough to explain the offset between the Pleiades and the field stars, which is of the order of $0.02 \mathrm{mag}$ in $\beta$ if we adopt the Hipparcos distance modulus of the Pleiades. In this connection we note that photometric observations of the $\beta$ index is quite straightforward; no extinction correction is needed and the transformation to the standard system is linear without color terms. As discussed by Olsen (1983), systematic differences in $\beta$ obtained with different telescopes and filter sets are 0.005 mag at most. Furthermore, we note that two of the Pleiades F-type stars from CP76 (Hz II 739 and 948) happen to be in Olsen (1999). The differences (Olsen - CP76) are 0.007 and 0.008 , respectively. This does not point to any large systematic errors, and a correction for this difference would in fact increase the offset of the Pleiades with respect to the field stars, but of course we cannot draw any strong conclusions from two stars only.

\subsection{Calibration and selection}

\subsubsection{Reddening}

To correct for reddening, the color excess is determined for individual stars as $E(b-y)=(b-y)-(b-y)_{0}$, where $(b-y)_{0}$ is found by an iterative calculation based on the empirical calibration given by Crawford (1975a):

$$
\begin{aligned}
(b-y)_{0}= & 0.222+1.11 \Delta \beta+2.7 \Delta \beta^{2}- \\
& 0.05 \delta c_{0}-(0.1+3.6 \Delta \beta) \delta m_{0}
\end{aligned}
$$

where

$\delta c_{0}=c_{0}-c_{0, \mathrm{ZAMS}}(\beta), \delta m_{0}=m_{0, \mathrm{Hyades}}(\beta)-m_{0}$,

$c_{0}=c_{1}-0.20 \cdot E(b-y), m_{0}=m_{1}+0.30 \cdot E(b-y)$,

and $\Delta \beta=2.72-\beta$. The standard relation between $\beta$, $m_{0, \text { Hyades }}$, and $c_{0, \text { ZAMS }}$ is found by interpolation between the data points given in Table I by Crawford (1975a). The iterative procedure uses the four Strömgren indices for each star, and an initial guess for the color excess as input data. The output (individual color excesses) was obtained when $E(b-y)$ converged at the 0.0001 level. The expected error for $E(b-y)$ is found as

$\sigma[E(b-y)]=\left[\sigma^{2}(b-y)+\sigma^{2}\left[(b-y)_{0}\right]\right]^{1 / 2}$

where

$$
\begin{aligned}
\sigma\left[(b-y)_{0}\right]= & {\left[\sigma^{2}(\Delta \beta)\left(1.11+5.4 \Delta \beta+3.6 \delta m_{0}\right)^{2}+\right.} \\
& \left.\sigma^{2}\left(\delta m_{0}\right)(0.1+3.6 \Delta \beta)^{2}+0.05^{2} \sigma^{2}\left(\delta c_{0}\right)\right]^{1 / 2}
\end{aligned}
$$

with $\sigma(\Delta \beta)=\sigma(\beta), \sigma\left(\delta m_{0}\right) \simeq \sigma\left(m_{1}\right)$, and $\sigma\left(\delta c_{0}\right) \simeq$ $\sigma\left(c_{1}\right)$. An estimate of this error is obtained by inserting the mean values of $\Delta \beta$ and $\delta m_{0}$ from the samples. For the Pleiades stars it is $\sigma[E(b-y)]_{\text {Pleiades }}=0.008 \mathrm{mag}$, while it for the field stars is $\sigma[E(b-y)]_{\text {Field }}=0.010$ mag.

\subsection{2. $[\mathrm{Fe} / \mathrm{H}]$ calibration}

To be able to select nearby field stars with the same metallicity as the Pleiades, $[\mathrm{Fe} / \mathrm{H}]$ is calculated for the Pleiades using the empirical calibrations of Nissen (1981). The calibration formula is as follows:

$[\mathrm{Fe} / \mathrm{H}]=-[10.5+50(\beta-2.626)] \delta m_{0}+0.12$

where $\delta m_{0}$ is like in Eq. (2), and the constant of 0.12 is the adopted $[\mathrm{Fe} / \mathrm{H}]_{\text {Hyades }}$. The adopted $[\mathrm{Fe} / \mathrm{H}]_{\text {Hyades }}$ does not affect the result of the comparison of the Pleiades with the field stars, because it is only relative. But the estimate of the absolute $[\mathrm{Fe} / \mathrm{H}]_{\text {Pleiades }}$ value is of course dependent on the assumed $[\mathrm{Fe} / \mathrm{H}]_{\text {Hyades }}$. The expected error in this calibration is obtained by a procedure similar to that presented in Sect. 2.2.1, but this time $\sigma\left(\delta m_{0}\right)=$ $\sigma\left(m_{0}\right)$ is used. The results are $\sigma([\mathrm{Fe} / \mathrm{H}])_{\text {Pleiades }}=0.07$ and $\sigma([\mathrm{Fe} / \mathrm{H}])_{\text {Field }}=0.07$.

\subsubsection{Reduction step by step}

The criterion for being an F-type star is set to be $2.59<$ $\beta<2.72$, which is the $\beta$ range used by Crawford (1975a) and Nissen (1981) for their calibration formulas (reddening and metallicity). 
To keep as many Pleiades members as possible, no stars from Table II (CP76) are rejected as a start except $\mathrm{Hz}$ II 948 which appear to be a non-member in both CP76 and the WEBDA database. For each member star the reddening is calculated by the procedure described in Sect. 2.2.1, and their photometry measurements are individually corrected. The mean reddening obtained is $\langle E(b-y)\rangle_{\text {Pleiades }}=0.031 \pm 0.004 \mathrm{mag}^{1}$, and the starto-star rms scatter is $S[E(b-y)]_{\text {Pleiades }}=0.022$ mag. Compared with the expected error of the $E(b-y)$ determination $\left(\sigma[E(b-y)]_{\text {Pleiades }}=0.008 \mathrm{mag}\right)$, this indicates significant star-to-star reddening differences across the cluster. The mean color excess for the Pleiades obtained here is in quite good agreement with former results obtained from other investigations, which in general are in the range of about 0.03-0.04 mag (e.g. Pinsonneault et al. 1998 used $E(b-y)=0.7 \times 0.04 \mathrm{mag} \simeq 0.03 \mathrm{mag}$; here using the relation between $E(b-y)$ and $E(B-V)$ from Crawford 1975b).

The reddening corrected $m_{0}$ values together with the $\beta$ observations are then used as input in Eq. (6), to get the Pleiades metallicity. The mean value derived is $\langle[\mathrm{Fe} / \mathrm{H}]\rangle_{\text {Pleiades }}=0.01 \pm 0.02^{1}$. This value is in very good agreement with spectroscopic results, which mostly come out with a near solar metallicity for the Pleiades (e.g. Boesgaard \& Friel 1990). The star-to-star rms scatter is $S([\mathrm{Fe} / \mathrm{H}])_{\text {Pleiades }}=0.13$, which is somewhat larger than the expected error $\left(\sigma([\mathrm{Fe} / \mathrm{H}])_{\text {Pleiades }}=0.07\right)$.

From the catalogue of field stars, used in this investigation, there are 12658 stars which have $2.59<\beta<2.72$ (thus F-type stars), but 1194 stars of this group do not have Hipparcos parallaxes, so the absolute magnitude could not be derived, and they are therefore rejected. For each star in the remaining sample the reddening is calculated, as described in Sect. 2.2.1, and the photometry of every star is individually corrected. Due to statistical fluctuations and a low mean reddening of the field star sample $(\langle E(b-y)\rangle=0.009)$ some stars turn out to have slightly negative values of $E(b-y)$. In order to avoid any bias these negative values were not changed. Finally, the reddening corrected $m_{0}$ values and the $\beta$ observations can be used as input data in Eq. (6), to derive the metallicity for every star.

We choose a metallicity range of $-0.10<[\mathrm{Fe} / \mathrm{H}]<$ 0.12 which is comparable to the Pleiades mean metallicity plus/minus a representative estimate of the metallicity scatter, and the number of $\mathrm{F}$-type field stars remaining in this interval is 3389. The mean $[\mathrm{Fe} / \mathrm{H}]$ of the remaining sample is not equal to $\langle[\mathrm{Fe} / \mathrm{H}]\rangle_{\text {Pleiades }}$ because the metallicity profile of the original field star sample peaks around $[\mathrm{Fe} / \mathrm{H}]=-0.15$, thus $[\mathrm{Fe} / \mathrm{H}]$ for the remaining sample is slightly shifted (by $0.01 \mathrm{dex}$ ) to a lower $[\mathrm{Fe} / \mathrm{H}]$. But since this is only half the error of the mean of the Pleiades $[\mathrm{Fe} / \mathrm{H}]$ the effect is ignored.

1 This value is based on all stars from Table II of CP76 except the likely non-members Hz II 739 and Hz II 948 (see Sect. 2.3).
An additional selection of the field stars is made on the basis of the relative parallax error. If the $M_{V_{0}}$ vs. $(b-y)_{0}$ diagram is considered, the error in the absolute magnitude $M_{V_{0}}$ of the field stars is affected by the errors in $V_{0},(b-$ $y)_{0}$, and the distance modulus (through $\pi$, the parallax). From the errors given in Sects. 2.1 and 2.2.1, the error in $V_{0}$ can be estimated as: $\sigma\left(V_{0}\right)=\left[\sigma^{2}(V)+(4.3 \sigma[E(b-\right.$ $\left.\left.y)]_{\text {Field }}\right)^{2}\right]^{1 / 2}=0.044 \mathrm{mag}$. The size of the effect on $\sigma\left(M_{V_{0}}\right)$ from $\sigma\left[(b-y)_{0}\right]$ depends on the slope of the ZAMS in the color region of interest. A test plot is made to find the approximate slope, and it is found to be $\sim 12$. The effect from $\sigma(\pi) / \pi$, on the distance modulus, is found by differentiating the relation $(m-M)=5 \log \left(\frac{1}{\pi}\right)-5$, with respect to $\pi^{-1}$, where $(m-M)$ is the distance modulus. This now leads to the following expression:

$\sigma\left(M_{V_{0}}\right) \simeq\left[\sigma^{2}\left(V_{0}\right)+\left(12 \sigma\left[(b-y)_{0}\right]\right)^{2}+(2.17 \sigma(\pi) / \pi)^{2}\right]^{1 / 2}$

The optimized choice of the upper limit of $\sigma(\pi) / \pi$, is when $2.17 \sigma(\pi) / \pi \sim \max \left\{\sigma\left(V_{0}\right), 12 \sigma\left[(b-y)_{0}\right]\right\} \sim 0.12 \mathrm{mag}$ (where $\sigma\left[(b-y)_{0}\right]_{\text {Field }}=0.010$ mag; Eq. (6)), which suggests $\sigma(\pi) / \pi \sim 0.05$. If a similar consideration is made in the $M_{V_{0}}$ vs. $(v-y)_{0}$ or $M_{V_{0}}$ vs. $\beta$ diagram, the suggestion would be $\sigma(\pi) / \pi \sim 0.04$. To avoid different samples of field stars in the three investigation parts, parallax measurements to a $5 \%$ accuracy are chosen, which left a sample of 782 stars. This seems to be the most reasonable choice, since the sample is large enough to make a clear definition of the ZAMS, and a lower error always is desirable.

\subsection{MS fitting analysis}

The MS fitting analysis is carried out in three diagrams $M_{V_{0}}$ vs. $(b-y)_{0}, M_{V_{0}}$ vs. $(v-y)_{0}$, and $M_{V_{0}}$ vs. $\beta$. Often only the $(b-y)_{0}$ case will be illustrated in the figures, but the results of the other diagrams will be given. Though $\beta$ is not a color, all three diagrams will in the following be denoted color-magnitude diagrams, and the specification "F-type" stars will be omitted, thus "the stars" or "all the stars" simply refers to the sample of F-type stars used in this investigation. A test plot of the Pleiades showed that there was an outlying star which was significantly cooler than the rest of the sample, and $\sim 1$ mag above the Pleiades MS (it has spectral type G0; Mendoza 1956). The star is rejected as a likely non-member. The Pleiades mean color excess and metallicity did not change significantly if the G0 star was included in the sample or not. The changes were only 0.002 mag in the average color excess, and $\langle[\mathrm{Fe} / \mathrm{H}]\rangle$ changed by 0.003 dex.

With the errors stated in Sects. 2.1 and 2.2.1 the estimated error per one star in $M_{V_{0}}$ for the field stars (Eq. (7)), is in the range of 0.14-0.17 mag for all three examined color-magnitude diagrams (smallest for the $(v-$ $y)_{0}$ diagram, and largest for the $(b-y)_{0}$ and $\beta$ diagrams, which is expected since the ZAMS in the $(v-y)_{0}$ diagram is less steep than in the other two diagrams).

In Fig. 1 the field stars are plotted together with the Pleiades stars adopting the Hipparcos mean parallax of 


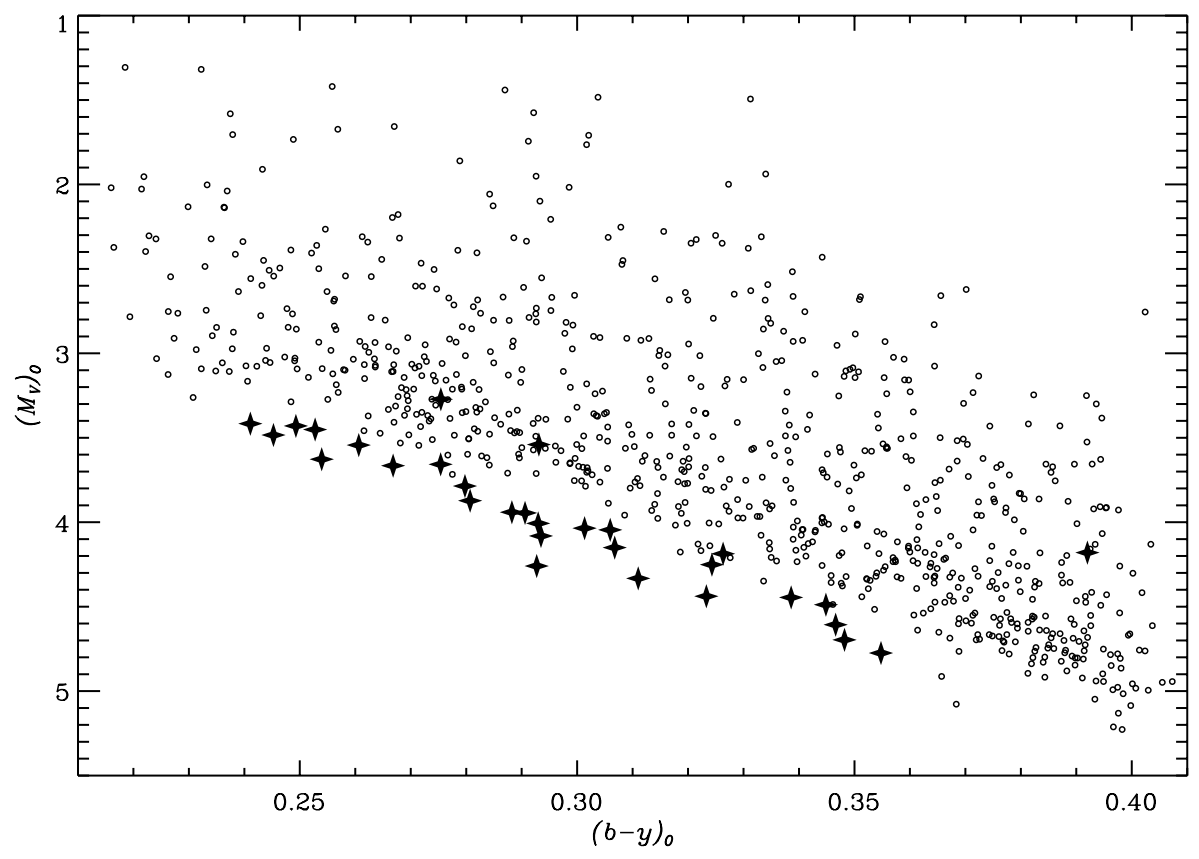

Fig. 1. Color-magnitude diagram of the selected field stars with $-0.10<[\mathrm{Fe} / \mathrm{H}]<0.12$ and $\sigma(\pi) / \pi<0.05$ (empty circles), compared to the Pleiades (filled stars) adopting the Hipparcos inferred distance modulus of 5.36 mag (from Robichon et al. 1999). The Pleiades star at the cooler end of the diagram is a G0 star and most likely a non-member, and the two stars (Hz II 1338 and 1912) about 0.6 mag brighter than the mean relation defined by the others Pleiades stars are binaries.

the cluster. This plot clearly shows the discrepancy between the locus of the field stars and that of the Pleiades. The plots in all three color-magnitude diagrams look quite similar. In addition to the deviating G0 star there are two Pleiades stars (Hz II 1338 and 1912) which are $~ 0.6$ mag brighter than the mean relation defined by the others. According to Mermilliod et al. (1992), one of the stars is a spectroscopic binary, and the other a visual binary, in agreement with their shift in brightness.

\subsubsection{The stellar magnitude distribution}

To get an estimate of the distance modulus of the Pleiades we have to analyze the magnitude distribution, relative to the Pleiades MS, of the field stars which define our zero point of the ZAMS locus. The observed magnitude distribution is a convolution of the underlying evolutionary distribution of the field star sample and a Gaussian profile coming from the observational errors in $M_{V_{0}}$.

A robust least squares fit (second-order) (Freudenreich 1999) of the Pleiades MS is made in the $V_{0}$ vs. color diagrams. The derived polynomial of the Pleiades MS is used to define the shape of the $\mathrm{ZAMS}_{\text {Field }}$. To find the distance modulus of the Pleiades the polynomial is shifted by the magnitude which make it fit to the $\mathrm{ZAMS}_{\text {Field }}$. The advantage of this method is that the robust least squares fit of the Pleiades MS will not be significantly affected by the stars which lie far from the Pleiades MS. This means that e.g. double stars will not bias the locus of the Pleiades MS significantly. The disadvantage is that the shifted Pleiades MS fit may not match the $\mathrm{ZAMS}_{\text {Field }}$ perfectly, because the shape of the fitted Pleiades MS is sensitive to the small sample of Pleiades data points (29 stars). The shifting of the Pleiades MS is done by calculating the individual distance moduli of every field star with respect to the polynomial fit of the Pleiades MS. This give the magnitude distribution of the field stars relative to the Pleiades MS. All the distance moduli are evaluated as input in the likelihood function which is the simultaneous probability function of all data points. The probabilities of the individual data points are described by the result of the convolution mentioned above. The underlying evolutionary distribution is approximated by $\propto \exp (-x / \tau)$ with a sharp edge at the $\mathrm{ZAMS}_{\text {Field }}$ locus, where $\tau$ is the fall-off rate due to evolution and binarity of the star sample. The width of the Gaussian is characterized by the observational error $\sigma\left(M_{V_{0}}\right)$. The mathematical expression of the convoluted function is:

$$
\begin{aligned}
f(u) & =\frac{p_{1}}{p_{2}} \exp \left(\frac{p_{4}^{2}}{2 p_{2}^{2}}-\frac{u-p_{3}}{p_{2}}\right) \frac{1}{\sqrt{2 \pi} p_{4}} \int_{-\infty}^{y} \exp \left(-\frac{v^{2}}{2 p_{4}^{2}}\right) \mathrm{d} v \\
& =\frac{p_{1}}{2 p_{2}} \exp \left(\frac{p_{4}^{2}}{2 p_{2}^{2}}-\frac{u-p_{3}}{p_{2}}\right)\left(1+\operatorname{Errf}\left(\frac{y}{\sqrt{2} p_{4}}\right)\right)(8)
\end{aligned}
$$

where $y=u-p_{3}-p_{4}^{2} / p_{2}, p_{1}$ is the normalization constant, $p_{2}=\tau, p_{3}$ is the ZAMS $\mathrm{ZAield}_{\text {locus, }} p_{4}=\sigma\left(M_{V_{0}}\right)$, and $\operatorname{Errf}(\mathrm{t})$ is the IDL error function. A best fit is obtained when the likelihood function takes its maximum value which we find by changing $\tau, \sigma\left(M_{V_{0}}\right)$, and the locus of the sharp edge of the $\mathrm{ZAMS}_{\text {Field }}$ as free parameters.

The underlying evolutionary distribution (dashed line) together with the convoluted function of best fit (thick line) is shown in Fig. 2. Additionally, the individual distance moduli are binned and over plotted to illustrate the 


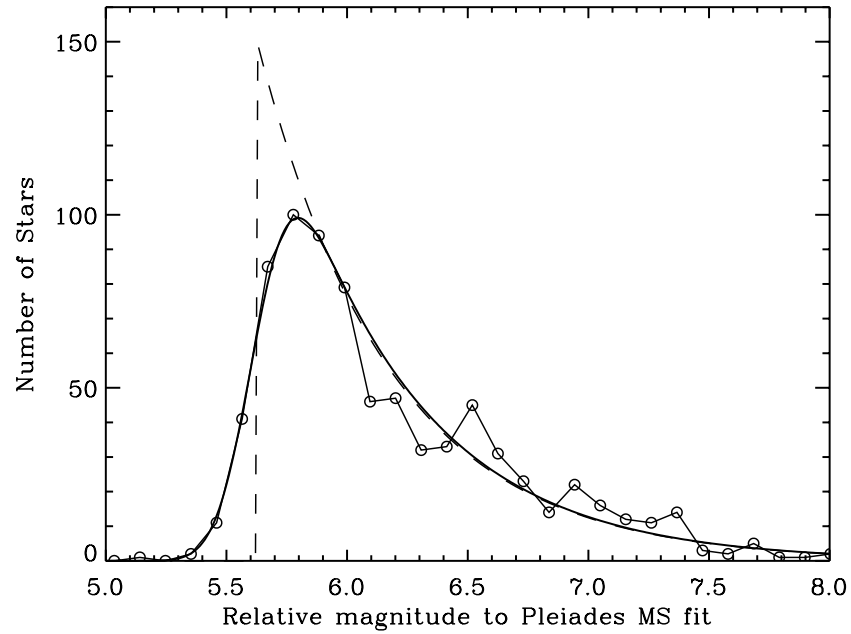

Fig. 2. The field star magnitude distribution, with respect to the polynomial fit of the Pleiades MS, in the $M_{V_{0}}$ vs. $(b-y)_{0}$ diagram. Empty circles shows the individual distance moduli binned, with a bin size of $0.1 \mathrm{mag}$ and connected with the thin line. Dashed line indicates the assumed underlying star distribution without observational errors defining the point of the $\mathrm{ZAMS}_{\text {Field }}$. The thick line is the best fit to the data of the convolution of the underlying star distribution with a Gaussian profile which corresponds to the observational error.

field star magnitude distribution for comparison (thin line and empty circles). The point of the $\mathrm{ZAMS}_{\text {Field }}$, is the vertical dashed line.

The fitted $\sigma\left(M_{V_{0}}\right)_{\text {Field }}$ for all three color-magnitude diagrams end up in the range $0.12-0.13 \mathrm{mag}$ which is near the expected errors derived in Sect. 2.3 on page 108. It indicates that the shape of the Pleiades polynomial matches the $\mathrm{ZAMS}_{\text {Field }}$ shape very well in all three color-magnitude diagrams. The Pleiades distance modulus found by the fits are: $5.62 \pm 0.02 \mathrm{mag}$, $5.61 \pm 0.02 \mathrm{mag}$, and $5.61 \pm 0.03 \mathrm{mag}$ in the $(b-y)_{0}$, $(v-y)_{0}$, and $\beta$ diagram respectively. The stated errors are the quadrature sum of two errors. The first comes from the uncertainty in the vertical positions of the Pleiades MS fits, which is the star to star scatter around the Pleiades MS fit divided by the square root of the number of stars $\left(S_{V_{0}}\right.$,Pleiades (Pleiades MS fit $\left.) / \sqrt{\# \text { stars }}\right)$. For the three color-magnitude diagrams these errors are: $0.019 \mathrm{mag}, 0.017 \mathrm{mag}$, and $0.031 \mathrm{mag}$ in the $(b-y)_{0}$, $(v-y)_{0}$, and $\beta$ diagram respectively ${ }^{2}$. The second error is found as the points where the log likelihood function has fallen by 0.5 from its maximum value by changing the Pleiades distance modulus step by step around its optimum value, and optimizing the two other parameters for every step. The $\pm 1 \sigma$ interval is approximated by a symmetric interval around the maximum value by a parabola fit of the log likelihood function. This approximation gives sufficient precision. For the three

\footnotetext{
2 These errors are from slightly around one to $3 / 2$ times larger than the pure observational scatter in $V_{0, \text { Pleiades, }}$ and show different real effects like binarity, which introduces extra scatter around the perfect ZAMS locus.
}

color-magnitude diagrams these errors are: $0.014 \mathrm{mag}$, $0.015 \mathrm{mag}$, and $0.015 \mathrm{mag}$ in the $(b-y)_{0},(v-y)_{0}$, and $\beta$ diagram respectively.

It is important that the left tail of the field star's magnitude distribution is fitted well because this is the region where the $\mathrm{ZAMS}_{\text {Field }}$ has to be found. The extremely good fit at the left tail of Fig. 2 supports the trustfulness of the method used in this investigation. The right tail is more affected by the evolutionary and binary distribution of the field star sample, and it could be argued that the assumed exponential fall-off at the right part of the profile is rather simplified, but changing it would not affect the fitted $Z_{\text {ZMS }}$ Field locus significantly because this part of the diagram is separated by several standard errors from the $\mathrm{ZAMS}_{\text {Field }}$ locus.

The method of finding the distance modulus presented here seems very robust because the distance moduli, errors in the distance moduli and $\sigma\left(M_{V_{0}}\right)$ are consistent with one another and their estimates in the three color-magnitude diagrams. By changing the underlying evolutionary distribution it is seen that the estimated distance moduli are quite stable. We consider the following different underlying star distributions. Assume the underlying fall-off to be in two steps. First a rather steep fall-off followed by a less steep fall-off. This scenario would fit the Gaussian to be wider (but could still be consistent with the estimates given in Sect. 2.3 on page 108), and the Pleiades distance modulus would be fitted to be slightly larger $\left(\mathrm{ZAMS}_{\text {Field }}\right.$ closer to observed maximum density). The only way to get a lower estimated distance modulus is by assuming a slower fall-off for the right tail of the underlying distribution; the extreme being a wide box function with one edge at the $\mathrm{ZAMS}_{\text {Field }}$. Under this assumption the $\mathrm{ZAMS}_{\text {Field }}$ will be at the point of half maximum of the observed distribution, which in this case means a lower distance modulus by less than 0.05 mag, but at the cost of an unacceptable bad fit to the data. One can of course get an even lower estimate if it is assumed that the underlying star density will increase on the right side of the $\mathrm{ZAMS}_{\text {Field }}$ in Fig. 2, which means that the maximum density of the underlying distribution is above the $\mathrm{ZAMS}_{\text {Field }}$. But the extremely good fits at the left tail (Fig. 2) and the consistent determinations of the Gaussian widths tell us that the underlying star distribution must have a sharp edge as indicated in Fig. 2. To see if the field star sample does indeed contain stars not evolved significantly away from the ZAMS, the sample is compared with a series of isochrones in Sect. 2.3.2, and the assumed underlying evolutionary distribution is tested by the aid of evolutionary tracks.

Figure 3 shows a plot similar to Fig. 1, but instead of the adopted Pleiades distance modulus of Hipparcos the estimated value of $5.62 \mathrm{mag}$, which make the fitted Pleiades MS polynomial match the $\mathrm{ZAMS}_{\text {Field }}$, has been used. Furthermore, the polynomial fit to the Pleiades MS is shown, shifted by $5.62 \mathrm{mag}$ (solid line) and by $5.36 \mathrm{mag}$ (dashed line). It is easy to see that the Pleiades shifted by 5.62 mag gives a much better fit to the field stars compared to the Hipparcos value. 


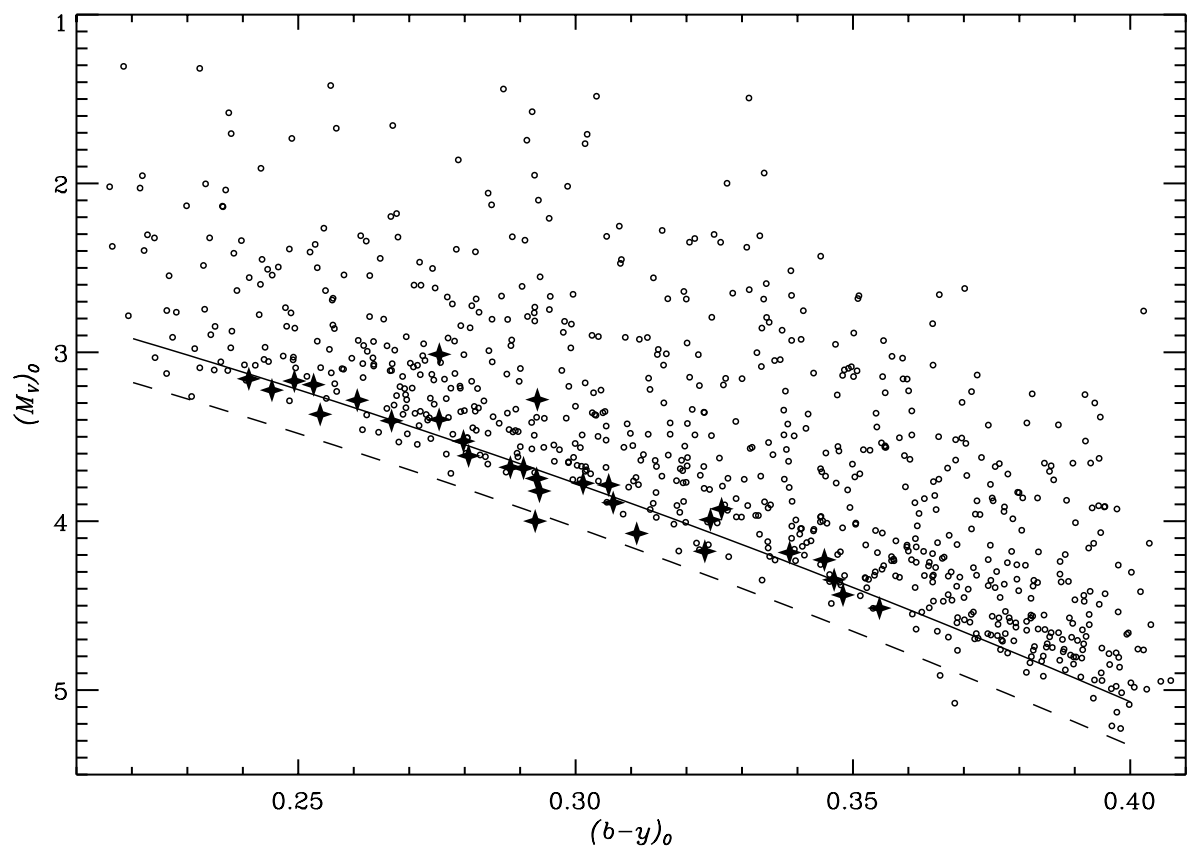

Fig. 3. Color-magnitude diagram of the selected field stars with $-0.10<[\mathrm{Fe} / \mathrm{H}]<0.12$ and $\sigma(\pi) / \pi<0.05$ (empty circles), compared to the Pleiades (filled stars) (excluding the likely non-member) adopting the distance modulus of 5.62 mag, which

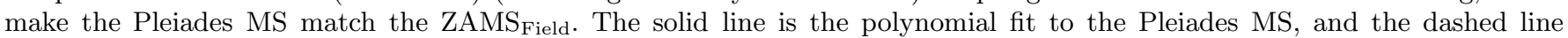
indicates the location of the Pleiades MS according to the Hipparcos distance.

\subsubsection{The age range}

The age of the field star sample which is used to determine the Pleiades distance modulus is analyzed by plotting isochrones of different ages together with the field stars.

The isochrones used are taken from Lejeune \& Schaerer (2001), those they denote as "basic grid" with solar metallicity. The effective temperature coming from the isochrones is transformed to the $(b-y)_{0}$ color index by the calibration of Alonso et al. (1996) using a mean value of the $c_{1}$ index in their Eq. (9). The possible systematic error in $(b-y)_{0}$ from this transformation is of the order of 0.02 mag.

Figure 4 shows the field stars in the color-magnitude diagram and five isochrones of ages: $100 \mathrm{Myr}, 500 \mathrm{Myr}$, 1 Gyr, 1.5 Gyr, and 2 Gyr. It is evident that the sample of field stars contains many stars with ages below or around 1.5 Gyr, which are all the stars at the left side of or around the 1.5 Gyr isochrone. Since we safely can assume that the age of the field stars is distributed uniformly in the plotted color range there must also be many field stars of ages around or less than 1.5 Gyr at the right side of the plot say in the range $0.30<(b-y)_{0}<0.40$. There may be a systematic offset of the isochrones with respect to the $\mathrm{ZAMS}_{\text {Field }}$ due to systematic errors in the $T_{\text {eff }}$ calibration, but Fig. 4 shows that the evolutionary effects on the isochrones from $100 \mathrm{Myr}$ (approximate age of the Pleiades cluster) to $1.5 \mathrm{Gyr}$ is rather negligible in the color range $0.30<(b-y)_{0}<0.40$. In that range we still see the discrepancy between the position of the Pleiades and the $\mathrm{ZAMS}_{\text {Field }}$ if the Hipparcos distance is adopted. Hence,

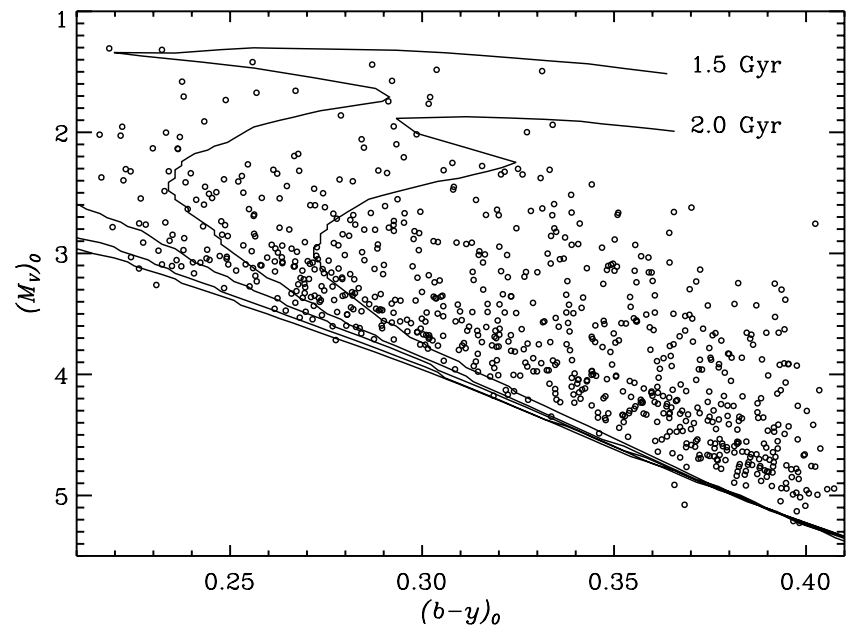

Fig. 4. Color-magnitude diagram of the selected field stars with $-0.10<[\mathrm{Fe} / \mathrm{H}]<0.12$ and $\sigma(\pi) / \pi<0.05$, compared to five isochrones representing the ages: $100 \mathrm{Myr}, 500 \mathrm{Myr}, 1 \mathrm{Gyr}$, 1.5 Gyr, and 2 Gyr.

we conclude that the discrepancy cannot be explained as due to evolution away from the ZAMS of the field star population.

As an additional check, evolutionary tracks from Lejeune \& Schaerer (2001) have been used to calculate the theoretical stellar magnitude distribution at a given $(b-y)_{0}$ in order to see how well it agrees with the underlying evolutionary distribution of $M_{V}$ adopted in Sect. 2.3.1. We assume a constant star formation rate for solar metallicity stars over the lifetime of the galactic disk ( $\sim 8 \mathrm{Gyr})$ in agreement with the age-metallicity diagram 
(Fig. 14) of Edvardsson et al. (1993), and a constant initial mass function over the small mass range corresponding to a given $(b-y)_{0}$. The calculated magnitude distribution is similar to the underlying evolutionary distribution of $M_{V}$ adopted in Sect. 2.3.1, i.e. with a sharp edge at the ZAMS and a steep evolutionary fall-off. The fall-off is less steep at the blue end of the $(b-y)_{0}$ range and somewhat steeper at the red end, and the fall-off has a tendency of a two-step function; first a steeper part followed by a less steep part. As discussed in Sect. 2.3.1 on page 110 this could indicate that our fitted Pleiades distance modulus is slightly underestimated. Altogether, we conclude that the assumed underlying evolutionary magnitude distribution of our field stars is supported by models for the stellar evolution.

\section{4. $[\mathrm{Fe} / \mathrm{H}]$ sensitivity of $Z A M S_{\text {Field }}$}

It is known that the ZAMS locus is dependent on the metallicity, such that low metallicity stars define a fainter ZAMS than the high metallicity stars. To be able to test if the discrepancies in the Pleiades distance could be explained as a possible error in the adopted Pleiades metallicity, we have analyzed how much the locus of the $\mathrm{ZAMS}_{\text {Field }}$ changes as a function of the metallicity.

The selected sample for this part of the investigation consists of all F-type field stars in the catalogue of Olsen (1999), which have a relative error in the parallax measurement less than $5 \%$. This selection gives a sample of 2309 stars. Five plots, each representing field stars in different metallicity intervals, are then made. The intervals are \pm 0.10 dex wide in $[\mathrm{Fe} / \mathrm{H}]$, ranging from -0.45 to +0.15 dex, and with a 0.10 dex overlap from one interval to the next.

Distance moduli for the Pleiades are found for the five metallicity intervals in all three color-magnitude diagrams. The method is similar to that presented in Sect. 2.3.1, and the individual distance moduli from every diagram is plotted together in Fig. 5. The indicated error bars in Fig. 5 are larger for the data points representing the lower metallicity intervals, which is a result of less stars falling in the lower metallicity bins. By analyzing the fits to the field star density profile of the lower metallicity intervals it was observed that the fitting function (see Sect. 2.3.1 Eq. (8)) did not fit the left tail of the distribution as well as shown in Fig. 2 simply because of the few data points available. Linear least squares fits are made to the result of all three color-magnitude diagrams in the metallicity range $-0.35<[\mathrm{Fe} / \mathrm{H}]<0.05$ (dashed lines), and additional fits where the two lowest metallicity points are ignored (solid lines).

The metallicity sensitivity from the three colormagnitude diagrams is found to be (solid lines):

$\Delta\left\langle M_{V_{0}}\right\rangle_{\mathrm{ZAMS}}=+0.01(9) \Delta[\mathrm{Fe} / \mathrm{H}]$, from $\beta$ $\Delta\left\langle M_{V_{0}}\right\rangle_{\text {ZAMS }}=-0.40(9) \Delta[\mathrm{Fe} / \mathrm{H}],(b-y)_{0}$ $\Delta\left\langle M_{V_{0}}\right\rangle_{\mathrm{ZAMS}}=-0.76(9) \Delta[\mathrm{Fe} / \mathrm{H}]$, and $(v-y)_{0}$.

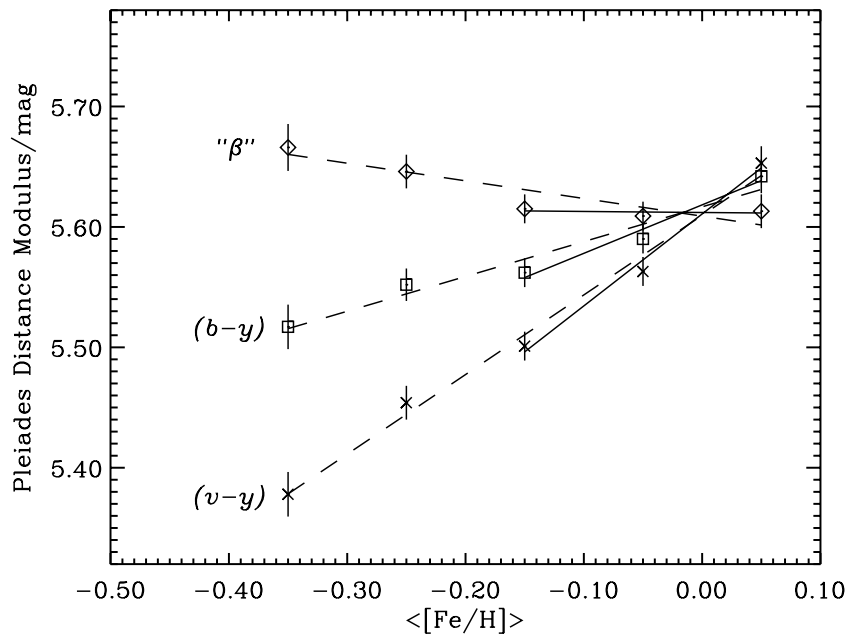

Fig. 5. Metallicity sensitivity of the ZAMSField in the three investigated color-magnitude diagrams: $\beta$ (diamonds), $(b-y)$ (squares), and $(v-y)(\times$ 's). Solid and dashed lines are least squares fits (see text).

The trend is the same for both solid and dashed lines, though solid lines show a higher metallicity sensitivity in $(b-y)_{0}$ and $(v-y)_{0}$ and no significant sensitivity in the $\beta$ diagram. We believe that the relations indicated by the solid lines are the most correct since the lower metallicity bins may suffer from small evolutionary effects due to a larger percentage of more evolved stars. That the highest metallicity sensitivity is observed in the $(v-y)_{0}$ diagram and the lowest in the $\beta$ diagram is expected since line blanketing from the metal lines affects the spectrum more at the short wavelengths, thus $v$ is more affected than $b$ which is again more affected than $y$. We note that Grenon (1999) finds a much larger shift $\Delta\left\langle M_{V_{0}}\right\rangle_{\mathrm{ZAMS}}=-1.67 \Delta[\mathrm{Fe} / \mathrm{H}]$ based on Geneva photometry, though the error of the metallicity sensitivity coefficient is not given. His relation refers, however, to early K-dwarfs and it is not clear on which color index it is based. A direct comparison can therefore not be made to the shifts we are finding for the F-type stars.

Figure 5 supports that the metallicity of the Pleiades is around the solar value as determined from the $m_{1}$ index if the adopted Hyades metallicity is $\sim 0.12$ dex. Only for this metallicity can we get consistent results of the distance modulus from all three color-magnitude diagrams. The inferred distance modulus is in the range 5.58-5.64 mag. We also see that a Hipparcos distance modulus of 5.36 mag is not consistent with one metallicity value. The $(v-y)_{0}$ diagram demands $[\mathrm{Fe} / \mathrm{H}] \sim-0.35$, the $(b-y)_{0}$ diagram $[\mathrm{Fe} / \mathrm{H}] \sim-0.70$, and the $\beta$ diagram cannot be fitted to a distance modulus of 5.36 mag no matter the chosen metallicity of the field stars.

From the above metallicity sensitivity analysis one could conclude that the $\beta$ diagram gives the most reliable estimate of the distance modulus, because it is rather insensitive to metallicity deviations between field stars and Pleiades stars, and furthermore it is insensitive to interstellar reddening. It should, however, be remembered that 
the $\beta$ diagram has the largest error in the distance modulus determination $(0.03 \mathrm{mag}) ; 3 / 2$ the size of the $(b-y)_{0}$ and $(v-y)_{0}$ diagram errors. If we combine the distance moduli from all three color-magnitude diagrams the final distance modulus is: $(m-M)_{0}=5.61 \pm 0.03 \mathrm{mag}^{3}$.

\section{Discussion and conclusion}

The extensive multi color MS fitting analysis based on Strömgren photometry (this paper) gives a Pleiades distance modulus of $5.61 \pm 0.03 \mathrm{mag}$ (the mean value from all three color-magnitude diagrams), which is in very good agreement with the distance modulus given by the multi color MS fitting analysis of Pinsonneault et al. (1998) who find a distance modulus of $5.60 \pm 0.05 \mathrm{mag}$. On the other hand, the distance modulus derived from the Hipparcos parallaxes are all in the range of 5.33-5.37 mag, which is not consistent with the former results, and the quoted errors. Even if the quoted errors from MS fitting are not representative for the actual uncertainty in this method, it must, from the current investigation, be concluded that the Hipparcos distance modulus is not consistent with the ZAMS of nearby field stars (Fig. 1). Thus, either are the Hipparcos parallaxes affected by systematic errors, which are significantly larger than expected, or there is something unexpected about the Pleiades cluster.

If the discrepancy is caused by some anomaly of the Pleiades, one possible explanation is the metallicity. Extensive investigation of this possibility has been performed (e.g. Pinsonneault et al. 1998 and the current investigation presented in Sect. 2.4), and from these results, supported by the spectroscopic metallicity determination by Boesgaard \& Friel (1990), it seems very unlikely that the adopted Pleiades metallicity around the solar value is more than 0.05 dex from the true value. Even a deviation of 0.1 dex is far too small to explain the discrepancy and it would lead to inconsistent results from the different colormagnitude diagrams. In addition, the relative comparison between the Pleiades and field stars of the same metallicity (Sect. 2) ensures that the possible difference in the relative metallicities are so small that a metallicity deviation can be ruled out. Hence, we cannot confirm the recent claim by Grenon (1999) that the Pleiades problem is solved by adopting a low metallicity, $[\mathrm{Fe} / \mathrm{H}]_{\text {Pleiades }}=-0.11$, of the cluster. Furthermore, the large metallicity difference between the Hyades and the Pleiades based on Geneva photometry, $\Delta[\mathrm{Fe} / \mathrm{H}]=0.25 \pm 0.03$, is inconsistent with the difference, $\Delta[\mathrm{Fe} / \mathrm{H}]=0.11 \pm 0.03$, which we derive from Strömgren photometry.

Another possibility is an abnormal helium abundance of the Pleiades. To see which value of $Y$ would be required to change the Pleiades MS locus by $0.3 \mathrm{mag}$, a calculation is made by Pinsonneault et al. (1998), who find that the

\footnotetext{
3 The distance modulus found by CP76, based on all 30 member stars in their Table II, was $(m-M)_{0}=5.53 \pm 0.04 \mathrm{mag}$, and by rejecting four stars, probably double stars or non-members, their result was $(m-M)_{0}=5.60 \pm 0.03$.
}

value is as high as $Y \simeq 0.37$. The study of Nissen (1974) revealed no intrinsic scatter in $Y$ greater than approximately $10 \%$ in nearby MS field B stars; much smaller than the $30 \%-40 \%$ change in $Y$ required for the Pleiades. There are, however, investigations which indicate large cluster to cluster scatter in the helium abundance (Nissen 1976; Lyubimkov 1977), and it has been suggested that this is the key explanation to the Hyades $c_{1}$-anomaly (Strömgren et al. 1982). To test if this is the explanation of the Pleiades problem too, an attempt should be made to measure the surface helium abundance of the hot stars in the Pleiades and other young clusters spectroscopically.

Recently, van Leeuwen (1999) has suggested that the Pleiades problem is caused by an age effect, and claims that other very young open clusters show the same deviation as the Pleiades. The investigation of van Leeuwen (1999) is based on a comparison in the color-magnitude diagram (with the metallicity-sensitive $B-V$ color) of nine open clusters, all shifted to their Hipparcos mean distance. But this is done without correcting for differences in the metallicity abundances first. A test of the age effect is made by Pinsonneault et al. (2000), based on 8 clusters (incl. the Hyades and Pleiades) and no age effect is seen in the difference between the MS fitting and Hipparcos distances. If the youth of the Pleiades has a significant effect on the Pleiades MS locus, one might also expect that young field stars would show the same effect. The study of Soderblom et al. (1998) of chromospherically active (and therefore assumed young) stars gave, however, no indications of that. In our own sample of field stars very few are expected to be as young as the Pleiades so we cannot test the suggestion of van Leeuwen (1999). It should be emphasized, however, that the explanation given by van Leeuwen (1999) of the Pleiades problem as an age effect, mostly relies on the relative shift between the Pleiades and Hyades in the temperature range where no obvious evolutionary effects away from the ZAMS are seen (corresponding to $(b-y)_{0}>0.30 \mathrm{mag}$ or $\left.(B-V)_{0}>0.50 \mathrm{mag}\right)$. Furthermore, the age explanation of van Leeuwen (1999) is in disagreement with theoretical models of stellar evolution, which predict negligible evolution away from the ZAMS during the first couple of billion years of the lifetime of late F-type stars (see isochrones in Fig. 4).

Some investigations give hints of spatial systematic errors in the Hipparcos parallaxes which are larger than expected (Pinsonneault et al. 1998; Narayanan \& Gould 1999). In addition, there is a statistical correlation caused by the imperfect distribution of data points over the ellipse described by the parallactic motion. In particular, for a star on the ecliptic, equal numbers of measurements should be obtained on both sides of the Sun. This was not fulfilled for Hipparcos, and caused correlations $\left(\rho_{\alpha}^{\pi}\right)$ between right ascension and parallax (ESA 1997, vol. 1, p. 325). Pinsonneault et al. (1998) questioned if this type of correlation would have an effect on the parallax values. But from the extensive test by Robichon et al. (1999) of this issue it must be concluded that the correlations 
$\rho_{\alpha}^{\pi}$ do not introduce significant errors in the Hipparcos parallaxes.

What if the whole controversy, about the Pleiades distance modulus, is caused by a real effect? What will the effects on the MS fitting and Hipparcos results be, if the cluster is non-spherical (sphericity has until now been implicitly assumed)? The angular size of the Pleiades cluster can be approximated by the size of the region covered by the member stars used in the investigation of e.g. Narayanan \& Gould (1999). This gives a radius of $\sim 6^{\circ}$ which, at a distance of $130 \mathrm{pc}$, corresponds to a radius of $\simeq 14$ pc. A typical real difference in the cluster member distances would then be around 14 pc which corresponds to a 1 mas difference in the parallax. There is no reason why non-sphericity of the Pleiades should not be the case. There is plenty of evidence that open clusters can be non-spherical e.g. NGC 2264 (the Cone Nebulae), and actually Raboud \& Mermilliod (1998) have shown that the distribution of the Pleiades stars projected on the sky is elliptical with an ellipticity of 0.17 . It could be that the cluster has a more oblong shape in the direction of the line of sight say with a length that is twice the projected diameter. One could then imagine the following scenario: the first born bright stars ( $\mathrm{O}$ and B-type) forms in one part of the gas cloud, and they start to blow the gas cloud in one initial direction, and therefore these stars will end up at one end of this deformed shape (as observed in the NGC 2264 case), and the fainter stars (F and G-type) will form a "tail" (as an overall trend). So if we see this shape head-on there will be a trend that the brightest B-type stars are closer to us, and the later classes are further away. Because the calculation of the Hipparcos mean parallax gives the largest weight to the brighter stars, the result will be a slightly shorter distance than the actual mean cluster distance (Pinsonneault et al. 1998, Fig. 20). On the other hand, the MS fitting method relies mostly on the fainter stars (A to G-type), which are farther away. These stars are located in the color-magnitude diagram, where the slope of the ZAMS is less steep, and therefore they give the smallest errors in the distance estimates (additionally for many clusters the hotter stars are also evolved away from the ZAMS). So this means that the distance found from MS fitting will be larger than found from Hipparcos parallaxes, and possibly closer to the actual mean cluster distance. Such oblong shape of the cluster, could indicate a kinematic history that does not follow the usual assumptions, which also explains why Robichon et al. (1999) find unusual features in the kinematics of the Pleiades. The consequence of this is that the assumptions used by Narayanan \& Gould (1999) does not hold. One could argue that if the Pleiades have such non-spherical shape, it would be expected that some fainter stars (which have larger distances) were present near the center of the cluster in e.g. Fig. 20 of Pinsonneault et al. (1998). But if there is a dark cloud just behind the bright stars as in the NGC 2264 case, none of these stars will be observed.

The idea of a deformed Pleiades cluster is a tempting answer to the Pleiades problem, because it includes most of the evidence presented in this discussion. What is described above, as a possible non-spherical Pleiades cluster, shall be viewed as an illustration or example of the possible effects on the distance determination, due to a deformed and non-symmetrical cluster. Though there are studies of the velocity dispersion among Pleiades cluster members (van Leeuwen 1994) and mass segregation (Raboud \& Mermilliod 1998) suggesting that the Pleiades is a bound and quite relaxed system, it could be very interesting to investigate the possibility of a non-symmetrical cluster by e.g. extensive kinematic analysis of the Pleiades. Furthermore, future astrometric space programs will be capable of determining the distances to the individual Pleiades stars with an improved accuracy of 2 to 3 orders of magnitude, compared with Hipparcos. These measurements will provide a very good three-dimensional picture of the Pleiades cluster.

Acknowledgements. We thank F. Grundahl for the merging of The Hipparcos Catalogue and the field stars catalogue, used in this investigation. This research has made use of the SIMBAD database, operated at CDS, Strasbourg, France.

\section{References}

Alonso, A., Arribas, S., \& Martinez-Roger, C. 1996, A\&A, 313, 873

Arenou, F., Mignard, F., \& Palasi, J. 1997, in The Hipparcos and Tycho Catalogues, vol. 3, ESA SP-1200 (Paris: ESA), 433

Boesgaard, A. M., \& Friel, E. D. 1990, ApJ, 351, 467

Crawford, D. L. 1975a, AJ, 80, 955

Crawford, D. L. 1975b, PASP, 87, 481

Crawford, D. L., \& Perry, C. L. 1976, AJ, 81, 419 (CP76)

Edvardsson, B., Andersen, J., Gustafsson, B., et al. 1993, A\&A, 275,101

Eggen, O. J. 1998, AJ, 116, 1810

European Space Agency 1997, The Hipparcos and Tycho Catalogues, ESA SP-1200 (Paris: ESA)

Freudenreich, H. 1999, The IDL Astronomy User's Library, http://idlastro.gsfc.nasa.gov/homepage.html

Grenon, M. 1999, in Proc. of 11th Cambridge workshop on cool stars, in press

Johnson, H. J., \& Mitchell, R. I. 1958, ApJ, 128, 31

van Leeuwen, F. 1994, in Galactic and Solar System Optical Astrometry, ed. L. V. Morrison, \& G. F. Gilmore (Cambridge University Press), 223

van Leeuwen, F. 1999, A\&A, 341, L71

van Leeuwen, F., \& Evans, D. W. 1998, A\&AS, 130, 157

Lejeune, T., \& Schaerer, D. 2001, A\&A, 366, 538

Lindegren, L. 1988, in Scientific Aspects of the Input Catalogue Preparation II, ed. J. Torra, \& C. Turon (Noordwijk: ESA), 179

Lindegren, L. 1989, in The Hipparcos Mission, ESA SP-1111, vol. III, ed. M. A. C. Perryman, et al. (Noordwijk: ESA), 311

Lindegren, L., Froeschle, M., \& Mignard, F. 1997, in The Hipparcos and Tycho Catalogues, vol. 3, ESA SP-1200 (Noordwijk: ESA), 323

Lyubimkov, L. S. 1977, Astrofizika, 13, 139 
Mendoza, E. E. 1956, AJ, 123, 54

Mermilliod, J.-C., Rosvick, J. M., Duquennoy, A., \& Mayor, M. 1992, A\&A, 265, 513

Mermilliod, J.-C., Turon, C., Robichon, N., Arenou, F., \& Lebreton, Y. 1997, in Hipparcos Venice 97, ed. B. Battrick, \& M. A. C. Perryman (Paris: ESA), 643

Mermilliod, J.-C. 2000, WEBDA-Database, http://obswww . unige.ch/webda/webda.html

Narayanan, V. K., \& Gould, A. 1999, ApJ, 523, 328

Nissen, P. E. 1974, A\&A, 36, 57

Nissen, P. E. 1976, A\&A, 50, 343

Nissen, P. E. 1981, A\&A, 97, 145

Olsen, E. H. 1983, A\&AS, 54, 55

Olsen, E. H. 1988, A\&A, 189, 173

Olsen, E. H. 1994, A\&AS, 106, 257

Olsen, E. H. 1999, Catalogue of uvby- $\beta$ photometry of $\sim 30000$ stars, private communication
Pinsonneault, M. H., Stauffer, J. R., \& Hanson, R. B. 1998, ApJ, 504, 170

Pinsonneault, M. H., Terndrup, D. M., \& Yuan, Y. 2000, ASP Conf. Ser. 198, Stellar Clusters and Associations, ed. R. Pallavicini, G. Micela, \& S. Sciortino, 95

Raboud, D., \& Mermilliod, J.-C. 1998, A\&A, 329, 101

Robichon, N., Arenou, F., Mermilliod, J.-C., \& Turon, C. 1999, A\&A, 345, 471

Robichon, N., Lebreton, Y., Turon, C., \& Mermilliod, J.-C. 2000, ASP Conf. Ser. 198, Stellar Clusters and Associations, ed. R. Pallavicini, G. Micela, \& S. Sciortino, 141

Soderblom, D. R., King, J. R., Hanson, R. B., et al. 1998, ApJ, 504,192

Strömgren, B., Olsen, E. H., \& Gustafsson, B. 1982, PASP, 94,5 